\title{
Whole-surface emission concentric shells for large light bulb using total internal reflection
}

\author{
Hiroshi Ohno ${ }^{*}$ and Mitsuaki Kato \\ Toshiba Corporation, Corporate Research and Development Center, Kanagawa, Japan
}

\begin{abstract}
Surface emission concentric shells that can emit both light rays and a large amount of heat from the whole outer surface are proposed here for a large globe light-emitting diode (LED) bulb. The concentric shells are composed of an outer total internal reflection (TIR) shell and an inner metallic shell adjacent to the outer shell. The TIR shell can guide light rays emitted from LEDs owing to the TIR and emits illuminating light from the outer surface. Assuming that the thickness of the TIR shell is sufficiently small, the TIR shell can also emit heat of the inner shell to the outside. It is theoretically predicted based on the heat transfer theory that the larger the concentric shells are, the larger the amount of heat that can be emitted to the outside. A large globe LED bulb prototype having the concentric shells with an outermost diameter of about $95 \mathrm{~mm}$ is therefore designed and fabricated, with which it is shown that an illuminating light flux of about 1064 lumens and heat of about $12.2 \mathrm{~W}$ can both be emitted to the outside. The prototype also shows to have a large hollow structure in which several kinds of devices can be accommodated. (C) The Authors. Published by SPIE under a Creative Commons Attribution 4.0 Unported License. Distribution or reproduction of this work in whole or in part requires full attribution of the original publication, including its DOI. [DOI: 10.1117/1.OE.60.8.085108]
\end{abstract}

Keywords: optics; geometrical optics; optical design; light-emitting diode; total internal reflection; bulb.

Paper 20210458 received Apr. 28, 2021; accepted for publication Aug. 3, 2021; published online Aug. 19, 2021.

\section{Introduction}

The replacement of traditional incandescent light bulbs with light-emitting diodes (LEDs) has been progressing rapidly owing to the high efficiency and long life of LEDs. The traditional incandescent light bulb, however, had some positive features such as a wide-angle light distribution with a half-intensity angle of more than $270 \mathrm{deg}$ and a simple appearance since it is covered by a transparent glass globe and has no metal heat sinks. Although the wide-angle light distribution is one of the most important features for lighting applications, the light distribution angle of the LED itself is not wide enough. Thus, additional optical elements are necessary to widen the light distribution angle. Several solutions have then been developed to produce wideangle light distributions. ${ }^{1-28}$

A total internal reflection (TIR) shell with LED light sources has also been developed, which can emit illuminating light from its surface. ${ }^{29}$ Concentric shells composed of the TIR shell that encloses a metallic inner shell are applicable to LED bulbs, enabling heat generated by the LED to be released through both the inner shell and the TIR shell to the outside. In Ref. 29, a standard pear-shape light bulb having the concentric shells with the outermost diameter of about $60 \mathrm{~mm}$ is fabricated, which is shown to emit an illuminating light flux of about 600 lumens.

Heat release rate from the concentric shells can be theoretically predicted to increase with respect to the outermost surface area of the concentric shells. The larger the TIR shell is, the larger the amount of heat that can be released to the outside. A large globe LED bulb composed of the concentric shells is therefore proposed with an outermost diameter of about $95 \mathrm{~mm}$, which can emit both light rays and a large amount of heat from the whole outer surface. The concentric shells are also advantageous in that several kinds of devices can be accommodated inside them. The remainder of this paper is organized as follows. First, the heat release rate with respect to the outermost diameter of the concentric shells is estimated based on the heat transfer theory.

*Address all correspondence to Hiroshi Ohno, hiroshi1.ohno@toshiba.co.jp 
Second, a design of the concentric shells for a large globe LED bulb prototype is described based on the geometrical optics. Third, the large globe LED bulb prototype is fabricated, which shows that an illuminating light flux of about 1064 lumens and heat of about $12.2 \mathrm{~W}$ can both be emitted to the outside. Lastly, discussions and conclusions are presented.

\section{Heat Release Rate of Concentric Shells}

Figure 1 shows a schematic cross-sectional view of concentric shells composed of an outer TIR shell and an inner metallic shell. The outer TIR shell is assumed to have a uniform thickness of $\delta$ with an outermost radius of $R_{\text {TIR }}$. Light rays emitted from LEDs will travel inside the TIR shell owing to the TIR. The inner metallic shell with an outermost radius of $R_{\mathrm{TIR}}-\delta$ is assumed to have sufficient thermal conductivity, which makes the temperature distribution uniform. Heat generated from the LEDs is thermally connected to the inner metallic shell. The heat will then be conducted through the TIR shell from the inner metallic shell and will be released to the outside by convection and thermal radiation.

The heat release rate, $Q_{\text {total }}[\mathrm{W}]$, from the concentric shells to the outside can be described based on the heat transfer equation. The temperature of the outermost TIR shell is set to $T_{\mathrm{TIR}}$ whereas that of the atmosphere (i.e., air) is set to $T_{\text {air }}$. Using a thermal convection resistivity $\Gamma_{\text {conv }}$ caused by the convection from the TIR shell with an emissivity $\varepsilon$ of the thermal radiation, the heat transfer equation can be written as

$$
Q_{\text {total }}=\frac{1}{\Gamma_{\text {conv }}}\left(T_{\mathrm{TIR}}-T_{\text {air }}\right)+4 \pi r_{\mathrm{TIR}}^{2} \varepsilon \sigma\left(T_{\mathrm{TIR}}^{4}-T_{\text {air }}^{4}\right),
$$

where the $\sigma$ denotes the Stefan-Boltzmann constant. The thermal convection resistivity $\Gamma_{\text {conv }}$ can be written with a heat transfer coefficient of natural convection, $\alpha\left[\mathrm{W} / \mathrm{m}^{2} / \mathrm{K}\right]$, as

$$
\Gamma_{\text {conv }}=\frac{1}{4 \pi \alpha R_{\mathrm{TIR}}^{2}} .
$$

Figure 2 shows the heat release rate of $Q_{\text {total }}[\mathrm{W}]$ calculated using Eq. (1) with respect to the outermost diameter of $2 R_{\mathrm{TIR}}$ of the concentric shells. Outermost diameters of standard-size and large-size bulbs are 60 and $95 \mathrm{~mm}$, respectively. The heat transfer coefficient of the natural convection, $\alpha$, is set to $10 \mathrm{~W} / \mathrm{m}^{2} / \mathrm{K}^{30}$ The $\varepsilon$ and $\sigma$ are set to 0.9 and $5.67 \times 10^{-8} \mathrm{~W} / \mathrm{m}^{2} / \mathrm{K}^{4}$, respectively. The outer surface temperature $T_{\mathrm{TIR}}$ of the TIR shell is set to $60^{\circ} \mathrm{C}$, which is less than the acrylic endurance temperature of $80^{\circ} \mathrm{C}$. The atmosphere temperature $T_{\text {air }}$ is set to $25^{\circ} \mathrm{C}$. It can be found that the heat release rate becomes more than $16 \mathrm{~W}$ if the outermost diameter is more than

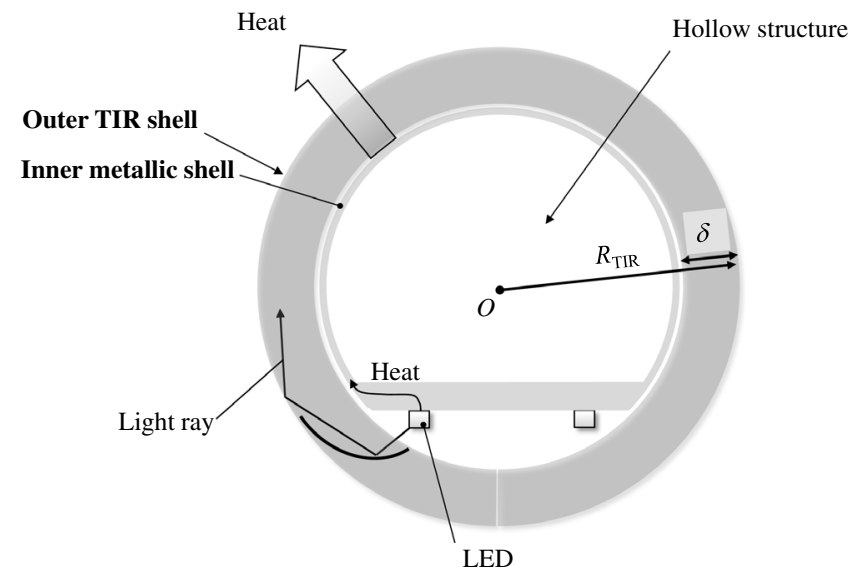

Fig. 1 Schematic cross-sectional view of concentric shells composed of outer TIR shell and inner metallic shell. The outer TIR shell is assumed to have a uniform thickness of $\delta$ with an outermost radius of $R_{\mathrm{TIR}}$. The inner metallic shell with an outermost radius of $R_{\mathrm{TIR}}-\delta$ is assumed to have sufficient thermal conductivity, which makes its temperature distribution uniform. 


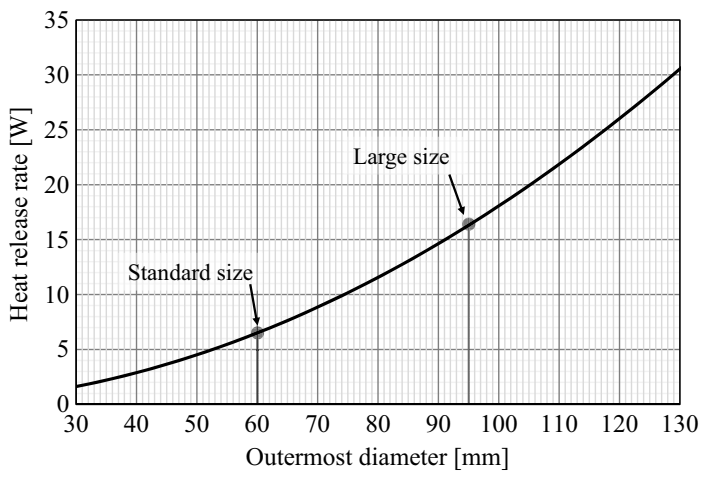

Fig. 2 Calculated heat release rate $Q[\mathrm{~W}]$ using Eq. (1) with respect to outermost diameter of $2 R_{\text {TIR }}$ of concentric shells. The outermost diameters of standard-size and large-size bulbs are 60 and $95 \mathrm{~mm}$, respectively.

that of the large-size bulb, namely, $95 \mathrm{~mm}$. On the other hand, the heat release rate becomes $<7 \mathrm{~W}$ if the outermost diameter is less than that of the standard-size bulb, namely, $60 \mathrm{~mm}$.

A temperature rise, $\Delta T_{\mathrm{TIR}}$, of the innermost TIR shell with respect to the outermost TIR shell can be written with the $Q_{\text {total }}$ represented by Eq. (1) and a thermal conductive resistivity $\Gamma_{\text {cond }}$ of the TIR shell as

$$
\Delta T_{\mathrm{TIR}}=\Gamma_{\mathrm{cond}} Q_{\mathrm{total}},
$$

where the $\Gamma_{\text {cond }}$ can be written with a thermal conductivity of the TIR shell, $\beta[\mathrm{W} / \mathrm{m} / \mathrm{K}]$, as

$$
\Gamma_{\text {cond }}=\frac{1}{4 \pi \beta}\left(\frac{1}{R_{\mathrm{TIR}}-\delta}-\frac{1}{R_{\mathrm{TIR}}}\right)
$$

Assuming that the TIR shell is made of acrylic, the thermal conductivity $\beta$ is set to $0.17 \mathrm{~W} / \mathrm{m} / \mathrm{K}$. The temperature rise $\Delta T_{\mathrm{TIR}}$ is about $12^{\circ} \mathrm{C}$ for the thickness $\delta$ of $3 \mathrm{~mm}$ with the $Q_{\text {total }}$ of $16 \mathrm{~W}$ and the $2 R_{\mathrm{TIR}}$ of $95 \mathrm{~mm}$. It can be found from Eqs. (3) and (4) that the thickness $\delta$ of the TIR shell should be as thin as possible to decrease the temperature rise of the $\Delta T_{\mathrm{TIR}}$ through reducing the thermal resistivity of the TIR shell.

In this work, the outermost diameter of the concentric shells is set to $95 \mathrm{~mm}$ with the TIR shell thickness $\delta$ of between 0.5 and $5.0 \mathrm{~mm}$, which enables a heat release rate about twice that of the standard-size bulb.

\section{Design of Concentric Shells for Large Globe LED Bulb}

\subsection{Basic Structure of Concentric Shells}

Figure 3 shows a schematic cross-sectional view of concentric shells composed of an outer TIR shell and an inner metallic shell for a large globe LED bulb with an outermost diameter of $95 \mathrm{~mm}$. The cross-sectional plane is set to $X-Z$ plane in a global Cartesian coordinate system $(X, Y, Z)$. The center of the concentric shells corresponds to the global coordinate origin $O$. The LEDs are axisymmetrically arranged around the $Z$ axis where their emitting surfaces face in the negative $Z$ direction.

Light rays emitted from the LEDs are guided by the TIR shell owing to the TIR toward the top of the LED light bulb in the positive $Z$ direction. In other words, the TIR shell can guide the light rays emitted from the LEDs in the negative $Z$ direction and gradually change the directions of the light rays toward the positive $Z$ direction. The inner surface of the TIR shell is coated with white light-scattering particles, which make the light rays traveling inside the TIR shell diffuse gradually to the outside. This allows the light rays to be emitted from the whole surface of the TIR shell to produce a wide-angle light distribution. The concentric shells also make it possible 


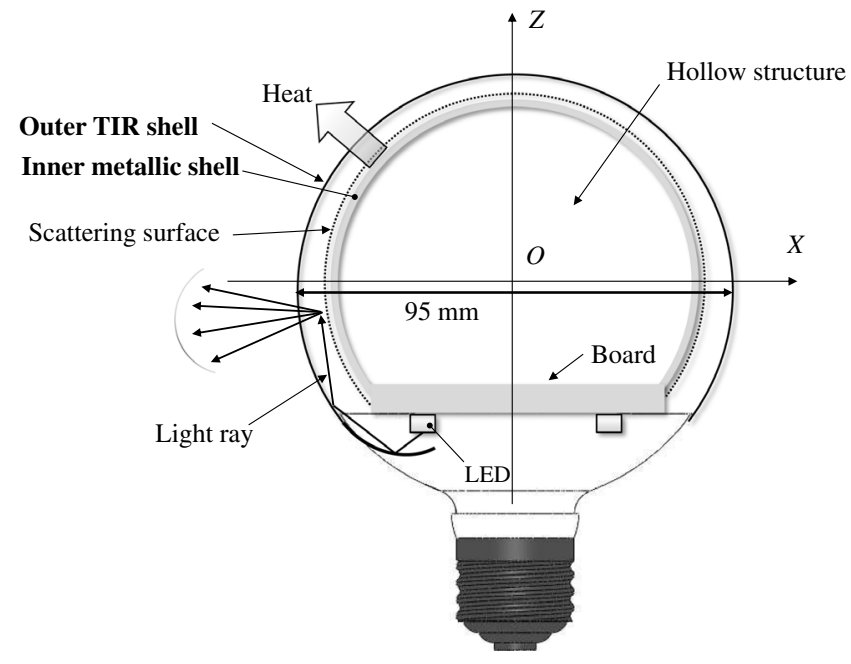

Fig. 3 Schematic cross-sectional view of concentric shells composed of outer TIR shell and inner metallic shell for large globe LED bulb with outermost diameter of $95 \mathrm{~mm}$. The cross-sectional plane is set to $X-Z$ plane in a global Cartesian coordinate system $(X, Y, Z)$. The center of the concentric shells corresponds to the global coordinate origin $O$. The LEDs are axisymmetrically arranged around the $Z$ axis where their emitting surfaces face in the negative $Z$ direction.

to release heat generated from LEDs to the outside from the whole outer surface. Furthermore, the concentric shells construct a hollow structure capable of accommodating several devices.

\subsection{Design of TIR Shell}

Figure 4 shows a schematic cross-sectional view close to an LED in $x-z$ plane of a local Cartesian coordinate system $(x, y, z)$ with its origin $O^{\prime}$ corresponding to an edge of the LED emitting surface. The $z$ axis is parallel to the $Z$ axis. A two-dimensional (2D) polar coordinate system $(\rho, \theta)$ is also taken with its origin corresponding to the $O^{\prime}$. The origin $O^{\prime}$ is located at distance of $\rho_{0}$ from the global coordinate origin $O$ in the direction of the $X$ axis.

A TIR curve that corresponds to the outer surface of the TIR shell reflects light rays coming from the edge of the LED (i.e., the origin $O^{\prime}$ ) owing to the TIR. A position vector $\boldsymbol{P}$ of the TIR curve is set to $(\rho, \theta)$. An infinitesimal displacement of the position vector, namely, $(\Delta \rho, \Delta \theta)$, satisfies the following equation based on the geometrical optics ${ }^{29}$ as

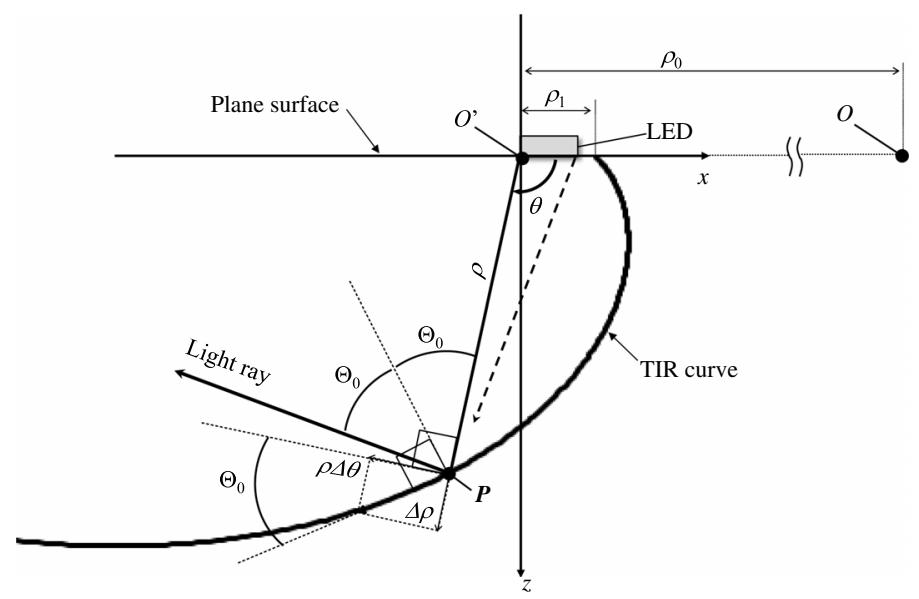

Fig. 4 Schematic cross-sectional view close to an LED in $x-y$ plane of a local coordinate system $(x, y, z)$ with its origin $O^{\prime}$. The origin $O^{\prime}$ corresponds to an edge of the LED emitting surface. A 2D polar coordinate system $(\rho, \theta)$ is also taken with its origin corresponding to the $O^{\prime}$. 


$$
\frac{\Delta \rho}{\rho \Delta \theta}=\tan \Theta_{0},
$$

where $\Theta_{0}$ represents an angle between the position vector $\boldsymbol{P}$ and an outward normal vector at the position. When the angle $\Theta_{0}$ is set to more than the critical angle $\theta_{c}$ of the TIR shell, a light ray emitted from the LED will always be reflected by the TIR at the position $\boldsymbol{P}$. The critical angle $\theta_{\mathrm{c}}$ can be written with the refractive index $n$ of the TIR shell as

$$
\theta_{c}=\arcsin \left(\frac{1}{n}\right)
$$

In this work, the angle $\Theta_{0}$ is set to a constant value that is a little larger than the critical angle of the acrylic. Equation (5) can thus be analytically solved as

$$
\rho=\rho_{1} \exp \left(\theta \tan \Theta_{0}\right),
$$

where $\rho_{1}$ denotes a radius of the local coordinate for $\theta=0$. When the $\rho_{1}$ is set to larger than a side length of the LED emitting surface, any light rays emitted from the LED can always be reflected by the TIR curve owing to the TIR because internal incident angles of the light rays on the TIR curve become larger than the critical angle of $\Theta_{0}$. This can be well described by the edge ray principle. ${ }^{31-34}$ Moreover, once a light ray is reflected by the TIR curve, the light ray consecutively continues to be reflected by the TIR curve because the internal incident angles of the consecutive reflections automatically become larger than the critical angle of $\Theta_{0}$. Any light rays emitted from the LED can therefore be guided along the TIR curve.

\subsection{Prototype of Large Globe LED Bulb}

A prototype of a light LED bulb having the large globe with diameter of $95 \mathrm{~mm}$ is designed using the concentric shells composed of the TIR shell and the metallic shell as shown in Fig. 5. In this work, the parameters of the TIR shell, namely, $\rho_{0}, \rho_{1}$, and $\Theta_{0}$ are set to $20.0 \mathrm{~mm}, 0.8 \mathrm{~mm}$, and $43 \mathrm{deg}$, respectively. The thickness $\delta$ of the TIR shell is set in a range of from 0.5 to $5.0 \mathrm{~mm}$. An axisymmetric LED array is composed of multiple LEDs with each emitting surface of $1.4 \mathrm{~mm} \times 2.8 \mathrm{~mm}$. The luminous efficiency of the LED is $85 \mathrm{~lm} / \mathrm{W}$ at operating temperature of about $80^{\circ} \mathrm{C}$. A supplemental mirror is introduced to reflect some of the light rays emitted from the LEDs into the TIR shell. A transparent flange is attached to protect the LEDs from dust.

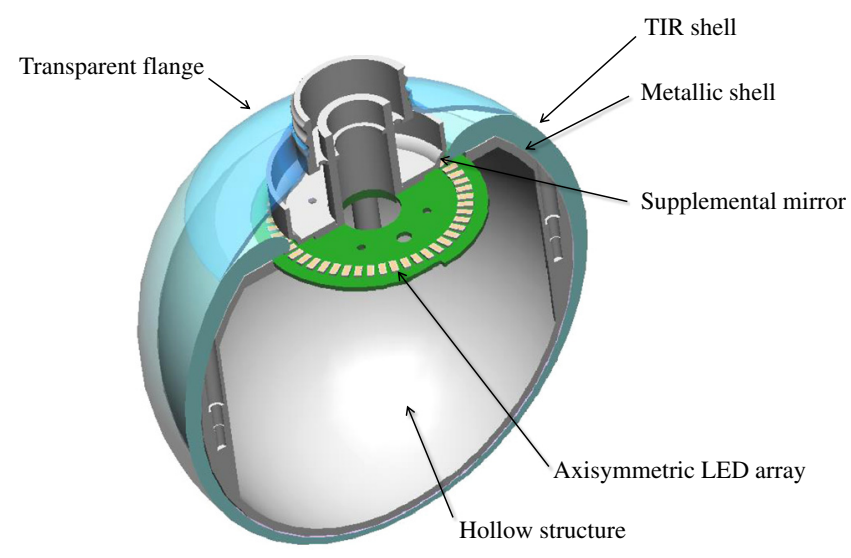

Fig. 5 Perspective view of half cut of light LED bulb prototype with large globe diameter of $95 \mathrm{~mm}$ using the concentric shells composed of TIR shell and metallic shell. Axisymmetric LED array is composed of multiple LEDs. Supplemental mirror is introduced to reflect some of the light rays emitted from the LEDs into the TIR shell. Transparent flange is attached to protect the LEDs from dust. Hollow structure can be constructed by concentric shells, which can accommodate several devices. 
A hollow structure can be constructed by the concentric shells, which can accommodate several devices.

\section{Experimental Results of Prototype of Large Globe LED Bulb}

The prototype of the large globe LED bulb composed of the concentric shells with outermost diameter of $95 \mathrm{~mm}$ is fabricated as shown in Fig. 6. The LED bulb turned off is shown in Fig. 6(a) and that turned on is shown in Fig. 6(b). A luminous flux is measured to be 1064 lumens at an input power of $16.0 \mathrm{~W}$ with an operating temperature of the LED of about $80^{\circ} \mathrm{C}$. The inner surface temperature of the TIR shell becomes about $65^{\circ} \mathrm{C}$ with atmosphere temperature of $25^{\circ} \mathrm{C}$ in the steady state. The heat release rate of the prototype to the outside is estimated to be $12.2 \mathrm{~W}$ where the rest of the power, $3.8 \mathrm{~W}$, is used for light ray generation. The heat release rate is larger than that of the standard-size LED bulb of about $7 \mathrm{~W}$ that is estimated in Sec. 2. Luminaire efficiency that is the ratio of light output emitted by the concentric shells to the light output emitted by the LED array is measured to be about $78 \%$.

Figure 7 shows a measured light angle distribution of the fabricated prototype of the large globe LED bulb where 0 deg corresponds to the positive $Z$-direction. Solid line denotes experimental result. The range of angle is from $-180 \mathrm{deg}$ to $0 \mathrm{deg}$ on the left and from 0 deg to +180 deg on the right. The half-intensity angle (i.e., half peak intensity angle range) is $310 \mathrm{deg}$.

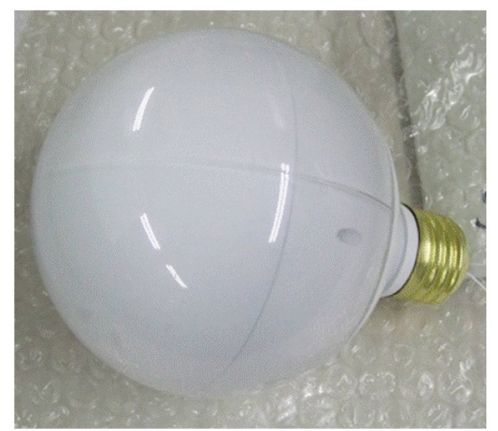

(a)

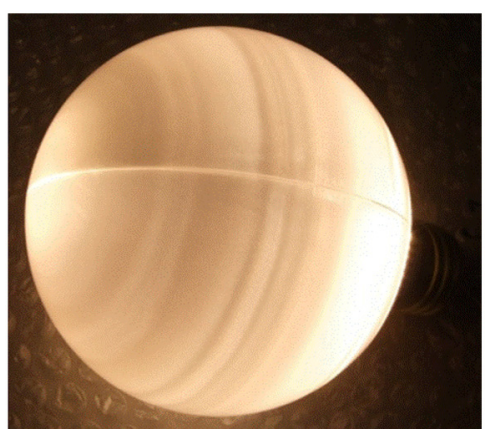

(b)

Fig. 6 Fabricated prototype of large globe LED bulb composed of the concentric shells with outermost diameter of $95 \mathrm{~mm}$. (a) The LED bulb turned off and (b) turned on.

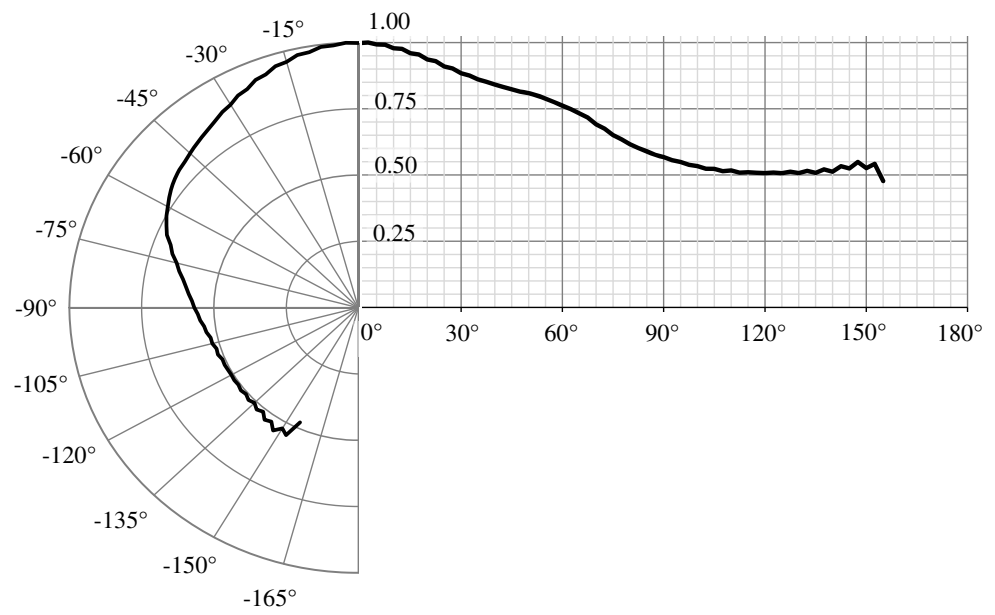

Fig. 7 Measured light angle distribution of the fabricated prototype of the large globe LED bulb. Solid line denotes experimental result. The range of angle is from -180 deg to 0 deg on the left, and from 0 deg to +180 deg on the right. 


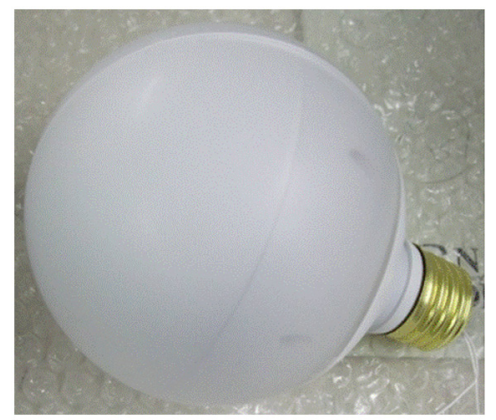

(a)

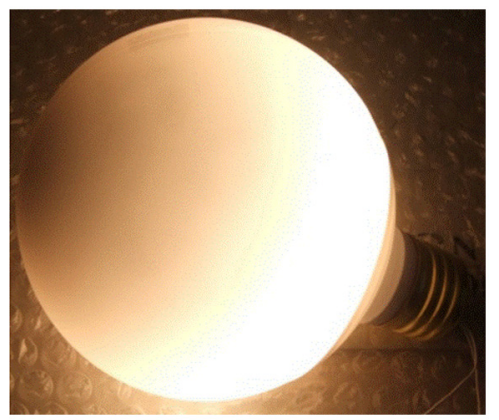

(b)

Fig. 8 Fabricated prototype of large globe LED bulb composed of concentric shells where the outermost surface is sandblasted. (a) The LED bulb turned off and (b) turned on.

Note that experimental data are measured in steady state with the operating temperature of the LED of about $80^{\circ} \mathrm{C}$.

\section{Discussions}

As shown in Fig. 5, the hollow structure constructed by the concentric shells could accommodate several devices, such as a brightness control circuit, a color control circuit, and an auxiliary battery. These devices would enhance the value of the LED bulb.

As shown in Fig. 6, a stripe illumination pattern is found on the LED bulb surface. The stripe pattern can be erased by sandblasting on the outermost surface of the LED bulb as shown in Fig. 8. The sandblasted LED bulb that is turned off is shown in Fig. 8(a) and that turned on is shown in Fig. 8(b).

The arrangement of the LED array is axisymmetric, and therefore the light angle distribution of the whole bulb should be axisymmetric as well. Although the light angle distribution shown in Fig. 7 is plotted only for one azimuth angle, the axisymmetric distribution is confirmed by both the experiment and the ray-tracing simulation. ${ }^{35}$

\section{Conclusions}

Surface emission concentric shells that can emit both light rays and a large amount of heat from the whole outer surface are proposed here for a large globe LED bulb with an outermost diameter of $95 \mathrm{~mm}$. The concentric shells are composed of an outer TIR shell and an inner metallic shell adjacent to the outer shell. The TIR shell can guide light rays emitted from LEDs owing to the TIR and emit illuminating light from the outer surface. Assuming that the thickness of the TIR shell is sufficiently small, the TIR shell can also emit heat of the inner shell to the outside. It is theoretically predicted using Eq. (1) that the larger the concentric shells are, the larger the amount of heat that can be emitted to the outside. A large globe LED bulb prototype having concentric shells with the outermost diameter of about $95 \mathrm{~mm}$ is therefore designed and fabricated, with which it is shown that an illuminating light flux of about 1064 lumens and heat of about $12.2 \mathrm{~W}$ can both be emitted to the outside through the whole outer surface. The heat release rate of the prototype of $12.2 \mathrm{~W}$ is larger than that of the standard-size LED bulb of about $7 \mathrm{~W}$. The measured light angle distribution of the prototype is wide enough where a half-intensity angle is $310 \mathrm{deg}$ as shown in Fig. 7. Furthermore, the prototype can have a large hollow structure in which several kinds of devices can be accommodated as shown in Fig. 5.

\section{Acknowledgments}

The authors would like to thank Tomoyuki Suzuki for valuable advice. The authors declare no conflicts of interest. 


\section{References}

1. H. Ohno, "Design of a coaxial light guide producing a wide-angle light distribution," Appl. Opt. 56(14), 3977-3983 (2017).

2. H. Ohno and T. Usui, "Gradient-index dark hole based on conformal mapping with etendue conservation," Opt. Express 27(13), 18493-18507 (2019).

3. Z. T. Ye, H. C. Kuo, and C. H. Chen, "Optical and thermal design of light emitting diodes omnidirectional light bulb," Appl. Opt. 54(28), E94-E101 (2015).

4. S. C. Allen and A. J. Steckl, "A nearly ideal phosphor-converted white light-emitting diode," Appl. Phys. Lett. 92, 143309 (2008).

5. J. K. Kim, T. Gessmann, and E. F. Schubert, "GaInN light-emitting diode with conductive omnidirectional reflector having a low-refractive-index indium-tin oxide layer," Appl. Phys. Lett. 88, 013501 (2006).

6. J. P. M. Ansems and J. H. A. Hagelaar, "Lighting device with omnidirectional light distribution," U.S. patent 2014/0252942 A1 (2014).

7. T. Kelly, T. Yeo, and D. Morin, "Light bulb with omnidirectional output," U.S. patent 8750671 B1 (2014).

8. C. Scianna and D. Plaines, "Omnidirectional lighting device," U.S. patent 6568834 B1 (2003).

9. T. Chen, "Light bulb with light emitting elements for use in conventional incandescent light bulb sockets," U.S. patent 7726836 B2 (2010).

10. P. Premysler, "Omnidirectional LED light bulb," U.S. patent 8680754 B2 (2014).

11. S. Liu and J. An, "Omnidirectional LED light bulb," U.S. patent 2014/0043823 A1 (2014).

12. N. Desilva, D. Nava, and R. Higley, "LED lamp with omnidirectional light distribution," U.S. patent 2014/0218931 A1 (2014).

13. Y. C. Chou, "Omnidirectional LED lamp and complex unitary lens," U.S. patent 8297799 B2 (2012).

14. N. Kanai, "Illuminating device," U.S. patent 8118457 B2 (2012).

15. D. Grabovickic, P. Benitez, and J. C. Minano, "TIR RXI collimator," Opt. Express 20(S1), A51-A61 (2012).

16. A. Rizkin and R. Beach, "LED light module for omnidirectional luminaire," U.S. patent 8360615 B2 (2013).

17. J. P. M. Ansems, V. S. D. Gielen, and M. J. Bosch, "Single chamber lighting device," U.S. patent 2013/0120965 A1 (2013).

18. C. M. Coushaine, "replaceable LED bulb with interchangeable lens optic," U.S. Patent 6637921 (2005).

19. K. Harrity et al., "Light apparatus," U.S. patent 7726860 B2 (2010).

20. P. J. Wu and P. C. Wu, "Structure of light bulb," U.S. patent 2010/0148650 A1 (2010).

21. K. H. Liu, "Illuminated traffic wand," U.S. patent 7261456 B2 (2007).

22. P. C. Chen, "Light guide tube and light emitting diode tube lamp incorporating the same," U.S. patent 2015/0029742 A1 (2015).

23. E. J. Tersa and B. Thibeault, "High output radial dispersing lamp using a solid state light source," U.S. Patent 6350041 (2002).

24. E. Boonekamp and T. Duester, "Light source," U.S. Patent 8292471 B2 (2012).

25. H. Ohno et al., "Lighting apparatus and light guide," U.S. Patent 2014/0362599 A1 (2014).

26. M. T. Lin et al., "Design of the ring remote phosphor structure for phosphor-converted white-light-emitting diodes," Jpn. J. Appl. Phys. 49, 072101 (2010).

27. H. Ohno, K. Nakagawa, and T. Kamikawa, "Design of secondary light source for reflectors with axisymmetric light guide," Appl. Opt. 58(14), 3848-3855 (2019).

28. H. Ohno, "Ghost secondary light source for LED collimated illumination," Appl. Opt. 59(33), 10339-10344 (2020).

29. H. Ohno and M. Kato, "Total internal reflection shell for light-emitting diode bulbs," Appl. Opt. 58(1), 87-93 (2019).

30. L. Cole et al., "A transient experiment to determine the heat transfer characteristics of a $100 \mathrm{~W}$ incandescent light bulb operating at 48 W," in Proc. Midwest Section Conf. American Society for Engineering Education (2012). 
31. J. Chaves, "Fundamental concepts," Chapter 1 in Introduction to Nonimaging Optics, pp. 3-23, CRC Press, New York (2008).

32. W. T. Welford and R. Winston, "Some basic ideas in geometrical optics," Chapter 2 in High Collection Nonimaging Optics, pp. 9-28, Academic Press, California (1989).

33. J. C. Minano, J. C. Gonzlez, and P. Benitez, "A high-gain, compact, nonimaging concentrator: RXI," Appl. Opt. 34, 7850-7856 (1995).

34. P. Benitez, J. C. Minano, and F. Munoz, "compact folded-optics illumination lens," U. S. Patent 7181378 (2005).

35. LightTools, https://optics.synopsys.com/lighttools/.

Hiroshi Ohno is a fellow at the Corporate Research \& Development Center of Toshiba Corporation. He received his BS, MS, and PhD degrees in physics from the University of Tokyo. His research interests include optics, photonics, nanotechnology, and cosmology with a special focus on Lagrangian optics, optical imaging, LED (light-emitting diode) lamp design, and high-power laser processes. He is a recipient of the 19th Japan Optical Design Award (2016), the 22nd Japan Optical Design Award (2019), the ODF'20 Best Paper Award (2021). He is a member of the Optical Society of Japan (OSJ), the Optical Society (OSA), and SPIE.

Mitsuaki Kato: Biography is not available. 